\title{
Testing Conditional Superannuation as an Anti-Doping Policy Supplement for Safeguarding Athlete Health and Welfare
}

\begin{abstract}
Given the significant, adverse health implications associated with performanceenhancing drugs in sport, anti-doping policy represents a pivotal intervention for not only protecting sport's credibility, but also for safeguarding athletes' health. However, current World Anti-doping Agency (WADA) policy has proven limited in controlling banned doping. This letter reports on a pilot experimental economics study testing 'conditional superannuation' as a novel anti-doping policy. A conditional superannuation policy compels athletes to direct a nominal percentage of their sportrelated earnings into a managed fund, and only returned to athletes after a period of retirement. Results demonstrate that the policy outperforms existing penalties such as fines and bans. With sport's hyper-competitive, commercial values, it remains improbable that an elite sports world will ever fully eliminate drugs. Nevertheless, sport needs regulation to help protect athletes from the health costs that arise from uncontrolled substance use. As existing penalty mechanisms appear to be inadequate for controlling sport doping, this letter recommends further research into conditional superannuation as a supplementary anti-doping policy to assist in safeguarding athlete health and welfare by lowering the incentive to utilize banned substances. The policy may also have applications to further domains of health including hospitals, allied health services, and aged care.
\end{abstract}

Keywords: Substance Abuse and Addiction; Regulation; Public Health, Sports 


\subsection{INTRODUCTION}

Faced with the implausible hope that all athletes will comply with sport's doping guidelines, the World Anti-Doping Agency (WADA) faces a seemingly insurmountable task. While $0.5-2.0 \%$ of doping control tests are returned positive, 'actual' rates of use have been reported at around 14-39\% of elite athletes (de Hon, Kuipers \& van Bottenburg, 2014), with the percentages significantly higher in some surveys (Moston, Engelberg \& Skinner, 2015). Former WADA Director General David Howman even conceded that some doping athletes could remain undetected throughout their careers. The broader issue associated with doping remains that their abuse can have serious, adverse consequences upon the health of users (Pope et al., 2013).

Under WADA's Anti-Doping Code, doping transgressions can incur various penalties typically focusing on disqualification and bans. Although penalties can include the relinquishment of winnings if proven that they were won while doping - as well as indirect consequences such as the loss of sponsorships-WADA's policy does not impose direct economic forfeits. In economic terms, athletes choose to dope when they estimate the health risk costs (plus the expected costs of penalties if caught) to be exceeded by the estimated marginal value accompanying improved performance (both pecuniary-earnings and sponsorships - and non-pecuniary-achievement, glory, adulation, etc.). In addition, in the event that less experienced athletes are more likely to test positive for doping than more the experienced, then transgression data reports an underestimation of the average age; a possibility encouraging the use of policies that better capture late-career athletes who might think they have less to lose from detection. In practice, conditional superannuation involves the evaluation of decision responses 
that cannot be accessed in any way other than through a research design accommodating hypothetical choices.

This letter summarizes a pilot study utilizing laboratory behavioral experiments with human subjects to test a novel, potential supplementary policy along the lines of a conditional superannuation (pension-fund-like) scheme. The policy mechanism works by compulsorily withholding a portion of athletes' earnings into a fund to be held in escrow and released after an interval following retirement, but only on the basis of an unblemished doping record (forfeited otherwise). Results from this study showed a decreased propensity for doping at no cost to athletes' efforts. Conditional superannuation proved to be a promising policy mechanism that might be used to supplement the existing WADA protocols.

This letter provides early evidence for an improved anti-doping policy mix, which could help reduce doping and better protect athletes' health, as well as their retirement welfare. In addition, the results augur favorably for the application of conditional superannuation to other forms of misconduct, such as match fixing or corruption, as well as to coaches, administrators and officials found complicit in overseeing or ignoring doping. In terms of the latter, some evidence demonstrates that athlete doping decisions are affected by their coaches and broader entourages (Huybers \& Mazanov, (2012; Mazanov et al., 2015). Given their heavily incentive-based and fiercely competitive salary structures, it is also plausible that senior executives or professional service practitioners could be subject to the mechanism as part of a remuneration structure designed to safeguard client and consumers against later revelations of 
maleficent conduct, negligence or abuse that occurred during their management oversight.

\subsection{METHODS}

\subsection{Design}

This pilot study began with the requirement for a research design employing hypothetical choices in order to test unavailable conditions. An experimental economics protocol was therefore adopted based on the design utilized by $\mathrm{Wu}$ et al. (2016). A total of 270 subjects (undergraduate students, as standard practice in pilot economic experiments) were asked — as hypothetical elite athletes with the potential to receive the rewards of competitive success - to make decisions about their training efforts, and whether to dope or not at various stages of their 'careers'. Subjects were offered incentives and required to respond regarding their training effort and whether to dope or not at different stages of their careers. The experimental conditions assumed that the subjects would seek to maximize their lifetime career earnings from a combination of prize money and endorsements. This maximization objective was reinforced via a 'real-world' reward in the form of cash paid to the subjects for part of their performances as hypothetical elite athletes. The rewards constituted the real-world equivalent of both prize money and endorsement income. Thus, the experiment was underpinned by a link between the real money a subject received and the virtual career earnings they accumulated. In addition, four different situational treatments made it possible to compare the effectiveness of different penalty schemes, thereby incorporating different detection probabilities for subjects to evaluate: no punishment, fines, bans, and conditional superannuation. 


\subsection{Experimental Model}

A parameterized model was employed, integrating a connection between decisions to dope and performance enhancement. Without any doping punishments, equilibrium emerges with all subjects choosing to dope in all periods. Each punishment (where $p$ is the fixed probability of a doper being caught) is variably effective, but the model predicts that bans in particular work less effectively on athletes approaching retirement. For the purposes of comparison, the model parameters (including $p$ ) equalized the impact (specifically, expected economic losses) of the three punishment regimes: bans, fines, and conditional superannuation.

There are $N$ heterogeneous (and risk-neutral) athletes where careers span $T$ years (a dynamic model). An athlete's performance (and their payoff), $q$, depends on three factors: i) underlying ability, $r$; and two choice variables: ii) their level of training effort, $e$; and iii) their (dichotomous) doping decision, $d$. An athlete starts with a (predetermined) raw talent level. Every period, an athlete's $r$ changes according to an age-dependent stochastic process (allowing a typical hump-shaped ability path) through his or her career. Changes in ability depend on the individual, the age, and the current ability of the athlete.

Following a non-competitive game as a primer, the competitive game was played in groups of three over three periods (analogous to Huck et al., 2011). Subjects' performances were linked to training effort and ability, which varied over time just as with any career. Subjects' training efforts were simulated by adding strings of single randomly generated digits, which became progressively longer (i.e. harder to solve), increasing the marginal cost of effort. Effort disincentives were combined with linear 
payoffs (i.e. identical reward for each sum solved correctly) and an extra payoff for unused time allotted (representing opportunity cost). It was therefore optimal for the subject to stop at some point, revealing the effort choice. A subject's athletic performance depended upon his or her choice to dope or not. Decisions to dope or not were made prior to task commencement, where the benefits (a performance boost, $\delta$ $>1$ ) and costs of doping were communicated via a text box message.

\subsection{RESULTS}

The results were generally in line with the theoretical predictions, in a similar sense to Macpherson et al. (2014) on "deferred compensation". They revealed that $87 \%$ of subjects decided to dope when it incurred no penalties. Conversely, penalties were effective in reducing doping probability for each of the three policies: bans $(31 \%$ doping probability), fines (18\%), and conditional superannuation (11\%). The effectiveness of the three penalties was compared using a standard nonparametric ranksum test. All proved significantly superior in lowering the probability of doping against the no-penalties benchmark, at 1\%. Fines and conditional superannuation (the latter at $1 \%$ ) performed better than bans. However, conditional superannuation did not diminish the probability of doping more than fines at any meaningful level.

Each penalty was also considered with respect to its impact on athlete effort exertion, since severity would undermine an athlete's cost-benefit evaluation. Athlete effort holds particular salience to anti-doping interventions because the most effective regulatory protocols discourage doping without undermining the incentive to compete. For the purposes of illustration, the most radical - if nonsensical—anti-doping regime might call for the death penalty, but its imposition may perversely eradicate participants 
unwilling to risk death in the event of a false positive doping test. Conversely, interventions perceived as being too weak by athletes might also reduce their efforts as success increasingly equates to doping vigor more than talent and training. In this study, bans (9.899) quantitatively returned the highest mean effort levels (i.e. number of additions attempted before quitting), followed by conditional superannuation (9.852), no punishment (9.350), and fines (8.899). No penalties, fines, and conditional superannuation all proved similar, but fines induced lower variations in effort between individuals. Significance-of-difference tests in athlete effort between the four alternatives revealed that each of conditional superannuation and bans could be respectively differentiated from fines (although merely at the $10 \%$ level).

In light of the balance between the doping choice risk / benefit calculation, the payoffs suggest that the subjects' decisions were most strongly affected by the conditional superannuation policy. Conditional superannuation delivered the most effective option when considered in terms of both doping probability and athlete effort jointly, since it outperforms bans in terms of lowering doping probability, and fines in terms of effort. Results were concordant with the Wu et al. (2016) theoretical model, although for some subjects in that study, doping was employed as a strategy to offset poor performance, rather than as a magnifier for increased training effort.

\subsection{DISCUSSION}

WADA's current policy imposes a differential penalty on transgressors, as the cost of bans and suspensions is lower for athletes close to retirement compared with those early in their careers. Although anecdotal, some sport authorities and commentators have hinted that older competitors might be more vulnerable to doping than their younger 
counterparts. Other studies have reported a correspondence between critical career transitions and the propensity to dope (Hauw \& Mohamed, 2015). If an age bias does hold, conditional superannuation offers a compelling addition to a policy mix skewed towards exclusion penalties. For example, a retrospective positive test of a stored sample during retirement could lead to the confiscation of some (or even all) of an athlete's accumulated funds.

Existing doping penalties are unlikely to exert an equivalent deterrent on all athletes, although little evidence has been collected concerning the differential effects of career stage and success. The most striking possibility is that the cost of risking a multiple year suspension is less for athlete close to retirement than for a new entrant. To speculate, the veteran athlete might be more attracted to doping in order to maintain competitiveness. Conversely, early-career athletes might be reluctant to risk interrupting their ascendance with a lengthy violation penalty. Coupé and Gerguad (2013) showed a 10\% positive correlation between cyclist age and the UCI Index of Suspicion of Doping, escalating to nearly $15 \%$ for riders over 30 . Other career timing evidence suggests that significant transitions and instabilities like serious injuries, performance slumps, and opportunities to compete at higher levels can increase vulnerability to doping (Erickson et al., 2013; Hauw \& Biland, 2012; Smith \& Stavros, 2020). As part of the policy mix, conditional superannuation could help athletes consider the implications of doping in the long-term context of their entire careers. For example, the policy could encourage athletes to avoid doping in order to to collect a post-retirement payout. Also, the policy allows for dopers to still be caught well after their transgressions when superior detection methods become available. 
Policy makers are faced with a significant challenge in working through anti-doping policy options, particularly if they are concerned as much with athletes' health as they are with fair play. Several advantages of integrating conditional superannuation (as an innovative incentive-based approach) into the existing policy are noteworthy, thereby offering a combination of both reward and deterrence. First, confiscated funds could be redeployed towards a range of preventative or remedial anti-doping initiatives such as education, or even on medical treatment. Second, athletes might benefit from a mandatory, professionally managed fund to support their post-sport lives (Carlson et al., 2015), which would be relevant to their long-term physical, psychological and social health. Third, the mechanism reduces the likelihood that a doping athlete will try to secure a big, one-off win and then retire with confidence, even if it means incurring serious drug-induced injury or illness as a result. Fourth, the policy guarantees that some portion of a doping athlete's unfairly gained returns are sequestered, potentially available for preventative strategies for the future. Finally, conditional superannuation could be applied to coaches and managers found guilty of orchestrating doping regimes, especially since athletes are susceptible to the influence of their entourage (Mazanov et al., 2015) who may privilege success over athlete health.

In terms of implementation, the policy would be centrally-administered by governing sporting authorities wherein athletes would have to forego a nominal percentage of prize money or income. The cumulative sum would sit in a (potentially) managed fund with the amount returned after a predetermined period of retirement during which no earlier samples re-tested positive. A positive test during an athlete's career or during the qualifying retirement period would result in the partial or full confiscation of the fund. The policy might be endorsed by athletes' unions. Resistance from athletes might 
be forthcoming, but like all WADA policies, athlete approval is not a prerequisite for adoption.

Notwithstanding the strongly held views that characterize the sport doping debate, there are many factors that make drug management in sport a vexing problem. There is no simple solution, and the fact that what may be considered a reasonable and appropriate drug use in a work, social or general health and medical setting, may not be appropriate in a sport related situation, further complicates the issue. Nor is it obvious that the doping problem can be cleared up with more targeted investigations, more frequent testing for even more drugs, or more punitive fines and bans.

Doping not only presents a significant regulatory issue for sport's authorities - and particularly WADA - it also imposes serious risks to athletes' health. Given that WADA's present policy seems to be sub-optimal, and by their own admission unable to eradicate doping, conditional superannuation might be considered as a promising policy supplement to the current Anti-Doping Code. This letter recommends that a credible case can be made for further research into conditional superannuation, such as a larger-scale replication of the current study using competing or retired athletes. It also suggests that conditional superannuation might be worthy of exploration as a policy mechanism in other health contexts.

\subsection{ACKNOWLEDGEMENTS}

$\mathrm{n} / \mathrm{a}$

\subsection{REFERENCES}


Carlson, K., Kim, J., Lusardi, A., \& Camerer, C.F. (2015). Bankruptcy rates among NFL players with short-lived income spikes. American Economic Review, $105(5), 381-384$.

Coupé, T., \& Gerguad, O. (2013). Suspicious blood and performance in professional cycling. Journal of Sports Economics, 14(5), 546-559.

de Hon, O., Kuipers, H., \& van Bottenburg, M. (2014). Prevalence of doping use in elite sports: A review of numbers and methods. Sports Medicine, 45(1), 57-69.

Erickson, K., McKenna, J., \& Backhouse, S.H. (2015). A qualitative analysis of the factors that protect athletes against doping in sport. Psychology of Sport and Exercise, 16(2), 149-155.

Hauw, D., \& Bilard, J. (2012). Situated activity analysis of elite track and field athletes' use of prohibited performance-enhancing substances. Journal of Substance Use, $17(2), 183-197$.

Hauw, D., \& Mohamed, S. (2015). Patterns in the situated activity of substance use in the careers of elite doping athletes. Psychology of Sport and Exercise, 16(20), 156-163.

Huck, S., Seltzer, A.J., \& Wallace, B. (2011). Deferred compensation in multiperiod labor contracts: An experimental test of Lazear's model. American Economic Review, 101(2), 819-843.

Huybers, T., \& Mazanov, J. (2012). What would Kim do?: A choice study of projected athlete doping considerations. Journal of Sport Management, 26(4), 322-334.

Macpherson, D.A., Prasad, K., \& Salmon, T.C. (2014). Deferred compensation vs. efficiency wages: An experimental test of effort provision and self-selection. Journal of Economic Behavior and Organization, 105(5), 90-107. 
Mazanov, J., Hemphill, D., Connor, J., Quirk, F., \& Backhouse, S.F. (2015). Australian athlete support personnel lived experience of anti-doping. Sport Management Review, 18(2), 218-230.

Moston, S., Skinner, J., \& Engelberg, T. (2012). Perceived incidence of drug use in Australian sport: A survey of public opinion, Sport in Society, 15(1), 64-72.

Pope Jr, H.G., Wood, R.I., Rogol, A., Nyberg, F., Bowers, L., \& Bhasin, S. (2013). Adverse health consequences of performance-enhancing drugs: An Endocrine Society scientific statement. Endocrine Reviews, 35(3), 341-375.

Smith, A. C., \& Stavros, C. (2020). Exploring the progressive use of performance enhancing substances by high-performance athletes. Substance Use \& Misuse, DOI: $10.1080 / 10826084.2019 .1711412,1-14$.

Wu, Q., Bayer, R.-C., \& Lenten, L.J.A. (2016). A comparison of anti-doping measures in sporting contests (Working Paper: 2016-11), University of Adelaide, School of Economics. 